\title{
WHAT GRAPHICAL AND STATISTICAL MATERIAL SHOULD BE INCLUDED IN THE NINTH-GRADE MATHEMATICS COURSE? ${ }^{1}$
}

\author{
By L. E. Mensenkamp, \\ High School, Freeport, Illinois.
}

The fact that the graph is a comparatively recent innovation in ninth-grade mathematics is well known. Not more than a decade ago there were in use in our secondary schools a number of algebras which made no use of it either in the elementary or the third-semester course. Other books of the same period accorded it some space in an appendix. In view of the fact that the graph is such a relatively new device in elementary. mathematics, it is not surprising to note a considerable variation in the amount and kind of treatment which it receives in the current texts.

It is our purpose here to examine the different uses to which the graph has been put in high-school mathematics in order to determine, if possible, just what portions of this material are suitable for the ninth-grade course. We must consider the precise motives which lie back of the teaching of each piece of graphical work. Material of this kind should be taught, not because it is the prevailing fashion to do so, but because each part of it has some definite contribution to make to the pupil's mathematical equipment. In selecting the classes of graphs which can best be studied in the first year course, it is also well to bear in mind the fact that a large proportion of the students of such a course will not go on to the work in advanced mathematics.

We shall consider the different phases of our topic in the order in which it seems, most desirable to have the student meet them in the classroom, that is, in the order of their relative difficulty. The type of graph which deals with the relations of certain empirical data is apt to have the strongest appeal to the beginner, and hence forms the most desirable introduction to the whole subject. Work of this kind has many possibilities because of the great variety of interesting statistical material there is to draw from. The pupil may plot data based on pricelevels, growth of population, weather reports, insurance, and a great diversity of economic and social phenomena. The local Y. M. C. A. may be running a membership campaign, and the boys will be greatly interested in recording its daily progress

1 Presented before the mathematics section of the Thirty-first Educational Conference of the University of Chicago, May $9,1919$. 
on squared paper or on the blackboard. The progress of the recent Liberty Loans in a community would have afforded excellent opportunities along the same line. Or, if practice exercises are being used in connection with the mathematics course, it may be well worth while to chart the attainments of the class from day to day. Because of the increasing use which is being made of graphs of this kind in our newspapers, magazines, and books, it is quite unnecessary to say anything to justify a consideration of them in the first-year course.

Not only should the pupil be taught how to set up statistical curves from tabulated data and how to read from them the value of one variable corresponding to a given value of the other, but he should also be required to consider the other things which they teach us. In connection with a curve showing the relation between the temperature and the time, for example, he should be required to locate the highest and the lowest temperatures; he should be required to tell at what time the temperature is rising or falling most rapidly, and in what intervals it is most nearly stationary. He will then begin to appreciate the striking advantages which the pictorial method of representation has over a table of numbers, as a means of exhibiting the essential relations between two changing quantities.

Of course it is possible to overdo all this. The enthusiastic teacher could doubtless spend a whole semester plotting such statistical curves, but it would be exceedingly unwise to go too far in this direction at the expense of some other equally important, if not so attractive, branch of mathematics.

After enough work of the kind just outlined has been carried out to make the pupil thoroughly familiar with the technique of the squared paper, that part of the subject more closely related to the traditional content of mathematics should be taken up. The transition should take place gradually through the plotting of such simple mathematical relationships as those which exist between the cost of a certain kind of goods and the amount purchased, the relation between the cost of a railway journey and the distance traveled, or the relation between the simple interest on a given amount of money and the time. The pupil should also construct formulas for the above relationships and thus be brought to see the connection between the algebra and the graph.

Next, problems may be taken up which require the use of two lines on the same paper. The following is an example: 
A has $\$ 12.00$ in the bank and starts to save at the rate of $\$ 3.00$ per week. B has no money in the bank; but at the same time as $A$, starts to save at the rate of $\$ 4.00$ per week. How long will it be until they have both saved the same amount? Here the money each has will be plotted against the time, and the solution will be found from the point on the time-axis corresponding to the intersection of the two straight lines. Many other problems can be devised which will depend upon the same method for their solution. The following is an illustration based on rates: A starts from a town at the rate of 5 miles per hour, and is followed 3 hours later by $B$, who proceeds at the rate of 7 miles per hour. How long will it take $B$ to overtake A? Here, again, the answer is obtained directly from the graph.

Many books give no space to problems of this kind, but there is much to commend them to the attention of the mathematics teacher. They deal with the graph directly in relation to some concrete problem, and the student is impressed with this new way of expressing mathematical relationships as he never could be if the work were confined to the somewhat mechanical procedure of plotting abstract equations. The meaning of the problem is kept in the foreground, and serves as a constant check against many of those mistakes in locating points which occur when students try for the first time to plot equations of the type $a x+b y=c$.

With the way thus carefully prepared, it is time to proceed to the more difficult matter of plotting pairs of simultaneous equations. The difficulties arise from a number of causes: Abstract equations are being plotted now; algebraic work of a more laborious nature is necessary to get the table of values; and negative numbers appear, making it necessary to consider all four quadrants of the plane.

Early texts often treated the graph as an isolated topic, but it is now recognized that it should be made an integral part of the exposition of the equation. It is clear that the main purpose of the graph here is not to serve as a device for the practical solution of equations-its defects for this purpose are obvious enough-but rather to interpret and give a geometrical meaning to such systems of equations and their solutions. When the pupil comes to associate the pairs of numbers which satisfy an equation in two unknowns with the points on a line, he sees at once why it requires two equations to obtain a unique 
solution in such cases; he sees in a vivid way why pairs of equations may sometimes occur which are incompatible; and he sees why still other pairs of equations may have an indefinite number of solutions. Any treatment of the graph which fails to make these connections absolutely clear to the student must be futile.

We have next to consider the employment of the graph of the quadratic equation in the first course. Textbooks are not at all unanimous in introducing it hère. It is not to be used as a means of solving the quadratic in one unknown, but rather as a means of interpreting geometrically the roots. From the graph the pupil perceives a new meaning in the fact that some quadratics have two real roots, others but one real root, and still others none at all. ${ }^{2}$ If the conception of the use of the quadratic graph just pointed out is a correct one, the question of whether or not it should be taken up in the first course would seem to resolve itself into the other question of whether or not we are justified in discussing the number and nature of the roots of the quadratic at this early stage in the pupil's career. Since most, if not all, of the quadratics which the freshman will have an actual need of solving will have two real roots, and since there are so many other more important topies in the usual crowded ninth-grade course which demand thoroughgoing treatment, it seems, on the whole, to be advisable to defer any discussion of the number of roots-and, consequently, the quadratic graph-until the advanced course.

The discussion of graphs in the first course may now be summarized as follows:

(1) The first graphs to be considered should deal with statistical material of a type which will appeal to the pupil.

(2) A number of simple mathematical graphs based on concrete problems (e. g., problems related to savings or rates) should then be taken up, and their solution obtained directly from the graph. The relation between the graph and the formula may be emphasized here.

(3) Graphs of simultaneous linear equations should be considered because of their value in giving a meaning to the solutions.

(4) Graphs of quadratics are not essential to the first course.

Any mention of equal roots or imaginary roots seems out of place in the first course, See the interesting remarks of Professor Huntington in Monographs on Modern Mathematics. edited by J. W. A. Young, pp. 153-155, also, p. I82. 